\title{
Holographic influence functional and its application to decoherence induced by quantum critical theories
}

\author{
Chen-Pin Yeh,,$* *$ Jen-Tsung Hsiang, ${ }^{1,2,+}$ and Da-Shin Lee ${ }^{1,+}$ \\ ${ }^{1}$ Department of Physics, National Dong-Hwa University, Hualien, Taiwan, R.O.C. \\ ${ }^{2}$ Center for Theoretical Physics, Fudan University, Shanghai, China
}

\begin{abstract}
The dynamics of a particle influenced by strongly-coupled quantum critical theories is studied by the holographic approach. A real-time prescription for the AdS/CFT correspondence in the context of nonequilibrium physics is proposed from mainly the field theoretic consideration, and the associated holographic influence functional is obtained. We then study the decoherence dynamics of a particle induced by the quantum critical theories with a dynamical exponent $z$. We find that as $z$ increases, the decoherence effect becomes less significant. The similar behavior is found as we heat up the environment. However, in this case the decoherence effect is enhanced not only by its strong coupling constant but also from the finite temperature effect. Finally a comparison is made with the result of a particle influenced by the free field.
\end{abstract}

PACS numbers: 11.25.Tq 11.25.Uv 05.30.Rt 05.40.-a

\footnotetext{
*Electronic address: chenpinyeh@mail.ndhu.edu.tw

${ }^{\dagger}$ Electronic address: cosmology@gmail.com

${ }^{\ddagger}$ Electronic address: dslee@mail.ndhu.edu.tw
} 


\section{INTRODUCTION}

Originally proposed as a holographic duality between the string theory and quantum field theory[1], AdS/CFT is found inspiring in understanding strongly coupled field theory. A well established dictionary is the relation between classical gravity action and the generating function for large $N$ quantum field theory (QFT) in the large coupling regime. This gives us a prescription to calculate the correlators of strongly coupled QFT by evaluating the corresponding classical gravity action. In particular, AdS/CFT correspondence has been applied to understand the strong coupling problems in condensed matter systems and the hydrodynamics(see [2, 3] for reivews). Independently in [4-6], it is also applied to explore the dissipation behavior of a particle moving in a strongly coupled environment. In these studies, the end point of the string on the boundary of the AdS black hole serves as a probe particle. Then more works are drawn to realizing the fluctuations of this end point of the string as Brownian motion in more general backgrounds [7-33]. For a general review on application in the non-equilibrium dynamics, please see [34].

From the aspect of the open systems in the field theory, the effects of environmental degrees of freedom on the particle can be accounted for by the method of Feynman-Vernon influence functional [35]. In this approach the environment variables are integrated out from the full density matrix to derive the evolution of the reduced density matrix with their effects encoded in the influence functional [37, 38]. This tracing-out process can be carried out by the path integral within the closed-time-path formalism (the so-called Schwinger-Keldysh formalism) [36]. However, usually the influence functional can at best be perturbatively calculated for a weakly coupled environment. The idea of the paper is to employ the holography method to find the influence functional of the strongly coupled environment.

We consider a probed particle as the system in a strongly-coupled quantum critical theory with general dynamical scaling $z$, in which the theory is invariant under the scaling,

$$
t \rightarrow \mu^{z} t, \quad x \rightarrow \mu x
$$

At zero temperature, the holographic description consists of a probed string moving in the classical Lifshitz geometry (see [27] and references therein) with the metric,

$$
d s^{2}=-r^{2 z} d t^{2}+\frac{d r^{2}}{r^{2}}+r^{2} d \vec{x}^{2}
$$


The holographic closed-time-path formalism is developed by Herzog and Son in [39] and later by Skenderis and Ree in [40]. We here extend the approach in [39] to the Lifshitz black hole background, but the correlators are obtained from the field-theoretic considerations. This prescription may provide a more transparent bridge in the AdS/CFT correspondence within the context of nonequilibrium field theory.

As an application, we use the holographic influence functional to study the quantum decoherence in a strongly coupled environment. Quantum decoherence is an ubiquitous phenomenon owing to an unavoidable interaction between the system of interest and the environment that has a huge number of the degrees of freedom. Recently many efforts have been devoted to the experimental realization of quantum computers, in which the central obstacle is to prevent the degradation of the quantum coherence of the computer by the disturbance from the environment [41]. This environment-induced effect has been studied in the framework of quantum open systems [42-45]. For example, the quantized electromagnetic fields can result in the stochastic motion of the classical charged particle [46-48]. Additionally they give rise to decoherence effects on the wavefunction of the quantum charged particle [43-45]. The effects can be observed in the interference pattern through the phase shift and the contrast change of the fringes as the consequence of quantum fluctuations of electromagnetic fields. One of the main focuses of this paper is to study how the interference of the particle states are affected by strongly coupled environment in its vacuum and thermal states.

Our presentation is organized as follows. In next section, we introduce the closed-timepath formalism. By tracing out the environment degrees of freedom in the full density matrix of the system and environment, we obtain the reduced density matrix of the system. In the linear response region, the environment effects are encoded in the associated influence functional. The holographic influence functional is then constructed with the prescription on the dual theory of a probed string in the classical Lifshitz geometry in Sec. III. In Sec. IV], we apply the obtained influence functional to study quantum decoherence of a particle in quantum critical theories. This reduction of the interference is measured by the decoherence functional, see [45]. Concluding remarks are in Sec. V. 


\section{CLOSED-TIME-PATH FORMALISM}

In the context of nonequilibrium physics, it is often convenient to use the so-called closedtime-path formalism (Schwinger-Keldysh formalism). Here we express the overall effects of the environment by the path integral along a closed time contour in the complex time plane, and then the expectation value for the system states, in particular, the nonequilibrium correlators can be obtained accordingly. To see it, let us consider that $\hat{\rho}(t)$ is the density matrix of the system-environment, and then it evolves unitarily according to

$$
\hat{\rho}\left(t_{f}\right)=U\left(t_{f}, t_{i}\right) \hat{\rho}\left(t_{i}\right) U^{-1}\left(t_{f}, t_{i}\right)
$$

with $U\left(t_{f}, t_{i}\right)$ the time evolution operator of the whole system. We then assume that the initial density matrix at time $t_{i}$ can be factorized as

$$
\rho\left(t_{i}\right)=\rho_{q}\left(t_{i}\right) \otimes \rho_{F}\left(t_{i}\right)
$$

where $q$ and $F$ are system and environment variables respectively. Initially the environment field $F$ is in thermal equilibrium at temperature $T=1 / \beta$ with the density matrix $\rho_{F}\left(t_{i}\right)$ given by taking the form

$$
\rho_{F}\left(t_{i}\right)=e^{-\beta H_{F}}
$$

where $H_{F}$ is the Hamiltonian for the environment field. The initial vacuum state with zero temperature can be reached by taking $T \rightarrow 0$ limit.

After we trace out the environmental degrees of freedom in the full density matrix $\hat{\rho}$, the environment effect on the system of interest is then realized in the reduced density matrix $\rho_{r}$. The reduced density matrix $\rho_{r}$ is given by

$$
\begin{aligned}
\rho_{r}\left(q_{f}, \tilde{q}_{f}, t_{f}\right)= & \int d \tilde{F}\left\langle q_{f}, \tilde{F}\left|\rho\left(t_{f}\right)\right| \tilde{q}_{f}, \tilde{F}\right\rangle \\
= & \int d \tilde{F} \int d q_{1} d F_{1} \int d q_{2} d F_{2}\left\langle q, \tilde{F}\left|U\left(t_{f}, t_{i}\right)\right| q_{1}, F_{1}\right\rangle \\
& \times\left\langle q_{1}, F_{1}\left|\rho\left(t_{i}\right)\right| q_{2}, F_{2}\right\rangle\left\langle q_{2}, F_{2}\left|U^{-1}\left(t_{f}, t_{i}\right)\right| \tilde{q}, \tilde{F}\right\rangle \\
= & \int d q_{1} d q_{2} \int d \tilde{F} d F_{1} d F_{2} \int_{q_{1}}^{q_{f}} \mathcal{D} q^{+} \int_{q_{2}}^{\tilde{q}_{f}} \mathcal{D} q^{-} \int_{F_{1}}^{\tilde{F}} \mathcal{D} F^{+} \int_{F_{2}}^{\tilde{F}} \mathcal{D} F^{-} \\
& \times \int_{F_{2}}^{F 1} \mathcal{D} F^{\beta} \exp \left[i \int_{t_{i}}^{t_{f}} d t L\left[q^{+}, F^{+}\right]-L\left[q^{-}, F^{-}\right]\right] \\
& \times \exp \left[i \int_{t_{i}}^{t_{i}-i \beta} d t L_{F}\left[F^{\beta}\right]\right] \rho_{q}\left(q_{1}, q_{2}, t_{i}\right) .
\end{aligned}
$$




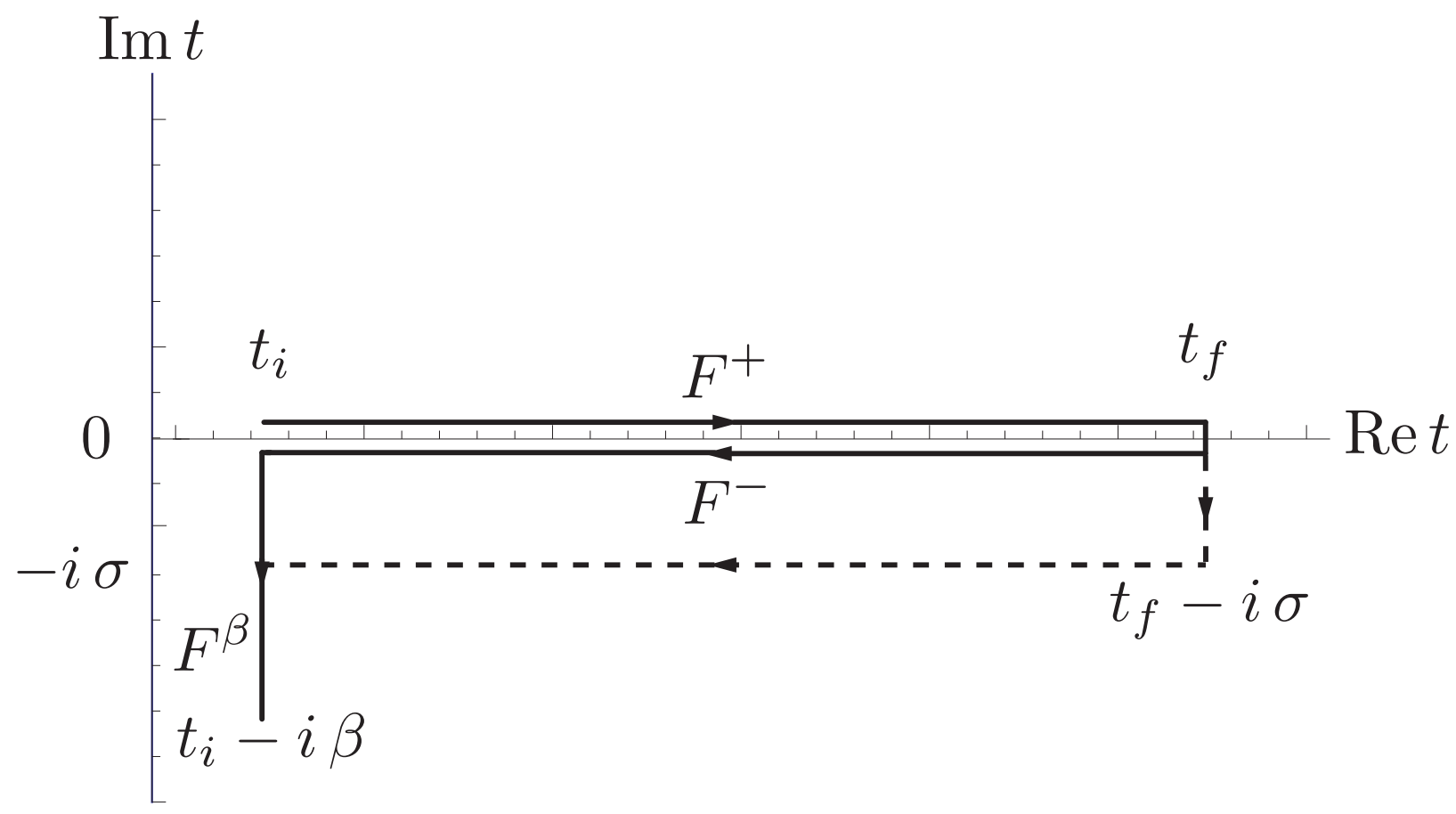

FIG. 1: The contour $(C)$ of the closed-time-path formalism (Solid line). The variable field labeled by $+(-)$ lives on the upper (lower) time axis, while the field labeled by $\beta$ lives along the imaginary time axis. The contour can be generalized to the finite $\sigma$ depicted in (Dotted line).

Here we have inserted the complete set of eigenstates, $\left|q_{1}, F_{1}\right\rangle$ and $\left|q_{2}, F_{2}\right\rangle$, which are given by the direct product of the system and environment states. The matrices of time evolution operators, $U\left(t_{f}, t_{i}\right)$ and $U^{-1}\left(t_{f}, t_{i}\right)$ can be expressed by the path integral along the forward and backward time evolution, represented by $q^{+}, F^{+}$, and $q^{-}, F^{-}$, respectively. The density matrix for the thermal state of the environment corresponds to the evolution operator matrix of the field $F^{\beta}$ along a path between the complex time $t_{i}$ and $t_{i}-i \beta$ (see Fig. 1). Thus, the Green's functions of the field possess the periodicity as the result of the cyclic property of the trace as well as the bosonic nature of the field operator.

We consider the system linearly coupled to an environment field $F$. The full Lagrangian is

$$
L(q, F)=L_{q}[q]+L_{F}[F]+q F .
$$

We write the evolution of the reduced density matrix in the form

$$
\rho_{r}\left(q_{f}, \tilde{q}_{f}, t_{f}\right)=\int d q_{1} d q_{2} \mathcal{J}\left(q_{f}, \tilde{q}_{f}, t_{f} ; q_{1}, q_{2}, t_{i}\right) \rho_{q}\left(q_{1}, q_{2}, t_{i}\right)
$$


where the propagating function $\mathcal{J}\left(q_{f}, \tilde{q}_{f}, t_{f} ; q_{1}, q_{2}, t_{i}\right)$ is

$$
\mathcal{J}\left(q_{f}, \tilde{q}_{f}, t_{f} ; q_{1}, q_{2}, t_{i}\right)=\int_{q_{1}}^{q_{f}} \mathcal{D} q^{+} \int_{q_{2}}^{\tilde{q}_{f}} \mathcal{D} q^{-} \exp \left[i \int_{t_{i}}^{t_{f}} d t\left(L_{q}\left[q^{+}\right]-L_{q}\left[q^{-}\right]\right)\right] \mathcal{F}\left[q^{+}, q^{-}\right]
$$

and the influence functional $\mathcal{F}\left[q^{+}, q^{-}\right]$is defined as

$$
\begin{aligned}
\mathcal{F}\left[q^{+}, q^{-}\right]= & \mathcal{N} \int d \tilde{F} d F_{1} d F_{2} \int_{F_{1}}^{\tilde{F}} \mathcal{D} F^{+} \int_{F_{2}}^{\tilde{F}} \mathcal{D} F^{-} \int_{F_{2}}^{F 1} \mathcal{D} F^{\beta} \exp \left\{i \int_{t_{i}}^{t_{i}-i \beta} d t L_{F}\left[F^{\beta}\right]\right\} \\
& \times \exp \left\{i \int_{t_{i}}^{t_{f}} d t L_{F}\left[F^{+}\right]+q^{+} F^{+}(t)-L_{F}\left[F^{-}\right]-q^{-} F^{-}(t)\right\} \\
= & \int d \tilde{F} \int_{C} \mathcal{D} F^{C} \exp \left[i \int_{C} d t L_{F}\left[F^{C}\right]+q^{C} F^{C}\right] / \int d \tilde{F} \int \mathcal{D} F^{\beta} \exp \left[i \int_{t_{i}}^{t_{i}-i \beta} d t L_{F}\left[F^{\beta}\right]\right] .
\end{aligned}
$$

In the end the influence functional can be expressed as a path integral along the contour $C$, as shown in Fig. 1, with the periodical boundary condition $F^{C}\left(t_{i}\right)=F^{C}\left(t_{i}-i \beta\right)=\tilde{F}$. The variables $F^{+}, q^{+}\left(F^{-}, q^{-}\right)$are associated with the upper (lower) branch of the closed-time contour while $F^{\beta}$ lives along the imaginary time axis. The normalization $\mathcal{N}$ has been chosen so that $\mathcal{F}[0,0]=1$. After carrying out the path integral over the environmental degrees of freedom, we find that

$$
\mathcal{F}\left[q^{+}, q^{-}\right]=\exp \left\{-\frac{1}{2} \int_{C \text { on } \pm} d t \int_{C \text { on } \pm} d t^{\prime}\left[q^{C}(t)\left\langle F^{C}(t) F^{C}\left(t^{\prime}\right)\right\rangle q^{C}\left(t^{\prime}\right)\right]\right\}
$$

where we have only kept the terms to the quadratic order of the system variable within the liner response region. The contour-ordered Green's functions are defined as

$$
\left\langle F^{C}(t) F^{C}\left(t^{\prime}\right)\right\rangle=\left\langle F(t) F\left(t^{\prime}\right)\right\rangle \theta_{C}\left(t-t^{\prime}\right)+\left\langle F\left(t^{\prime}\right) F(t)\right\rangle \theta_{C}\left(t^{\prime}-t\right),
$$

where the $\theta_{C}$ function is defined according to the path ordering along the contour. The correlators are defined with respect to the thermal state of the environment field. The periodicity boundary condition in the path integral along the contour is a consequence of the thermal bath of the bosonic field

$$
\left\langle F^{C}\left(t_{i}\right) F^{C}\left(t^{\prime}\right)\right\rangle=\left\langle F^{C}\left(t_{i}-i \beta\right) F^{C}\left(t^{\prime}\right)\right\rangle
$$

leading to

$$
\left\langle F(t-i \beta) F\left(t^{\prime}\right)\right\rangle=\left\langle F\left(t^{\prime}\right) F(t)\right\rangle
$$


which obeys the Kubo-Martin-Schwinger condition. Expressed in terms of times at \pm branches, the influence functional $\mathcal{F}\left[q^{+}, q^{-}\right]$can be written in terms of real-time Green's functions,

$$
\begin{aligned}
\mathcal{F}\left[q^{+}, q^{-}\right]=\exp \left\{-\frac{i}{2} \int_{t_{i}}^{t_{f}} d t \int_{t_{i}}^{t_{f}} d t^{\prime}\right. & {\left[q^{+}(t) G^{++}\left(t, t^{\prime}\right) q^{+}\left(t^{\prime}\right)-q^{+}(t) G^{+-}\left(t, t^{\prime}\right) q^{-}\left(t^{\prime}\right)\right.} \\
& \left.\left.-q^{-}(t) G^{-+}\left(t, t^{\prime}\right) q^{+}\left(t^{\prime}\right)+q^{-}(t) G^{--}\left(t, t^{\prime}\right) q^{-}\left(t^{\prime}\right)\right]\right\}
\end{aligned}
$$

The various correlation functions are defined as

$$
\begin{aligned}
& i G^{+-}\left(t, t^{\prime}\right)=\left\langle F\left(t^{\prime}\right) F(t)\right\rangle, \\
& i G^{-+}\left(t, t^{\prime}\right)=\left\langle F(t) F\left(t^{\prime}\right)\right\rangle, \\
& i G^{++}\left(t, t^{\prime}\right)=\left\langle F(t) F\left(t^{\prime}\right)\right\rangle \theta\left(t-t^{\prime}\right)+\left\langle F\left(t^{\prime}\right) F(t)\right\rangle \theta\left(t^{\prime}-t\right), \\
& i G^{--}\left(t, t^{\prime}\right)=\left\langle F\left(t^{\prime}\right) F(t)\right\rangle \theta\left(t-t^{\prime}\right)+\left\langle F(t) F\left(t^{\prime}\right)\right\rangle \theta\left(t^{\prime}-t\right) .
\end{aligned}
$$

The path integral expression of the influence functional in (10) can be understood as the field propagator of $F^{ \pm}$with an source $q^{ \pm}$in their respective branches. However the field evolves unitarily, giving $\mathcal{F}[q, q]=1$ due to the fact that the real time propagators on the \pm branches cancel each other when setting $q^{ \pm}=q$. Thus, the unitarity property of the field evolution gives a relation among various Green's functions.

$$
G^{++}\left(t, t^{\prime}\right)+G^{--}\left(t, t^{\prime}\right)-G^{+-}\left(t, t^{\prime}\right)-G^{-+}\left(t, t^{\prime}\right)=0
$$

This relation can be checked straightforwardly from (16) in the real-time expressions. For the time-translation invariant states, the Fourier transform of the Green's function is defined as $G(\omega)=\int d t G(t, 0) e^{i \omega t}$, where the identity in (17) becomes

$$
G^{++}(\omega)+G^{--}(\omega)-G^{+-}(\omega)-G^{-+}(\omega)=0 .
$$

We will see later that the periodicity condition (14) and the unitarity property (18) of a bosonic thermal bath are fundamental requirements to construct the holographic influence functional.

\section{HOLOGRAPHIC INFLUENCE FUNCTIONAL}

In this section, we will employ the holographic method to derive the influence functional for the Brownian particle. The dual description of the particle coupled to quantum critical environment at finite temperature is the string propagating in the Lifshitz black hole. 
The idea [39] is to maximally extend the black hole geometry in the Kruskal coordinates, which has two asymptotic boundaries. By choosing appropriate boundary conditions for the perturbations of the string $Q(t, r)$ in this background geometry [39], the classical on-shell action of the string can be identified as the influence functional for the Brownian particle in the boundary theory

$$
\mathcal{F}\left[q^{+}, q^{-}\right]=S_{\text {gravity }}\left(Q^{+}\left(t, r_{b}\right), Q^{-}\left(t, r_{b}\right)\right)
$$

The variable $q(t)$ is the position of Brownian particle that corresponds to the end point of the string perturbation $Q(t, r)$, and $q^{ \pm}(t)=Q^{ \pm}\left(t, r_{b}\right)$. The parameter $r_{b}$ is a cutoff in the radial direction to render the action finite, and thus represents the location of the boundary. In accordance with the above closed-time-path formalism, $Q^{+}\left(t, r_{1}\right)$ and $Q^{-}\left(t, r_{2}\right)$ live in two respective regions with the different boundaries in the maximally extended black hole geometry. These two regions will be introduced later as shown in Fig. 2.

In the following, we will first introduce the maximally extended Liftshitz black hole and then solve the classical equation of motion for a probed string in this extended background. Instead of imposing the infalling boundary conditions at the horizons as in [39], we will propose an alternative approach, which makes use of the unitarity and periodicity properties of the bosonic thermal field theory, to construct the influence functional in (19). Thus we call it the "semi-holographic" approach. The holographic influence functional in [39] is rederived

in the Appendix B for comparison. However this "semi-holographic" approach provides a more transparent method to set up the correspondence between classical gravity theory and nonequilibrium strongly coupled quantum field theory. Along the same line of thoughts we expect that this approach will be straightforwardly used to obtain the holographic influence functional for more general initial states such as coherent or squeezed states [49], and also for the environmental degree of freedom that obeys the Fermi-Dirac statistics.

\section{A. Maximally extended Liftshitz black hole}

We consider the Brownian particle coupled to quantum critical theories at finite temperature [20, 27] in 3+1-dimension. The dual description is the string moving in the $4+1$ dimensional Lifshitz black hole geometry with the metric

$$
d s^{2}=-r^{2 z} f(r) d t^{2}+\frac{d r^{2}}{f(r) r^{2}}+r^{2} d \vec{x}^{2}
$$


where $f(r) \rightarrow 1$ for $r \rightarrow \infty$ and $f(r) \simeq c\left(r-r_{h}\right)$ near the black hole horizon $r_{h}$ with $c=(z+3) / r_{h}$. The detailed form of $f(r)$ is not relevant when we consider the low-frequency behavior of the perturbations. The corresponding temperature of the back hole and also of the boundary theory is

$$
\frac{1}{T}=\frac{4 \pi}{z+3} \frac{1}{r_{h}^{z}} .
$$

The Kruskal coordinates are used to describe the maximally extended geometry. It is convenient to first introduce a new radial coordinate $r_{*}$

$$
r_{*}=\int d r \frac{1}{r^{z+1} f(r)} .
$$

Notice that when $z>0$, we have $r_{*} \rightarrow-\infty$ as $r \rightarrow r_{h}$, and $r_{*}$ approaches a finite value $r_{*}^{\infty}$ as $r \rightarrow \infty$. The metric (20) then becomes

$$
d s^{2}=r^{2 z} f(r)\left(-d t^{2}+d r_{*}^{2}\right)+r^{2} d \vec{x}^{2}
$$

The Kruskal coordinates $(U, V)$ are defined as

$$
V=e^{2 \pi T\left(t+r_{*}-r_{*}^{\infty}\right)}, \quad U=-e^{-2 \pi T\left(t-r_{*}+r_{*}^{\infty}\right)} .
$$

The product, $U V=-e^{4 \pi T\left(r_{*}-r_{*}^{\infty}\right)}$, depends only on the radial coordinate $r$. The exterior of the black hole is the region $V \geq 0, U \leq 0$, where $U V=-1$ as $r \rightarrow \infty$ and $U V=0$ as $r=r_{h}$. This is called region $I$ (see Fig. 2 for details). Notice that near the horizon $r_{h}$ :

$$
r_{*} \simeq \frac{r_{h}^{-z}}{z+3} \ln \left(\frac{r}{r_{h}}-1\right) .
$$

Thus $U V \propto \frac{r}{r_{h}}-1$ and it changes sign as one moves from the black hole exterior to the interior. We can analytically continue the coordinates to either $U>0, V>0$ or $U<0, V<0$ which corresponds to the black hole interior. However here we are interested in another continuation that also describes the black hole exterior with $U>0, V<0$, called region II . Both region $I$ and II contain the time-like infinity at $r \rightarrow \infty$, where the boundary theory can be defined. We label the spatial coordinate in region $I$ as $\left(\vec{x}_{1}, r_{1}\right)$ and in region $I I$ as $\left(\vec{x}_{2}, r_{2}\right)$. This will be the spacetime structure for which the holographic influence functional is formulated. 


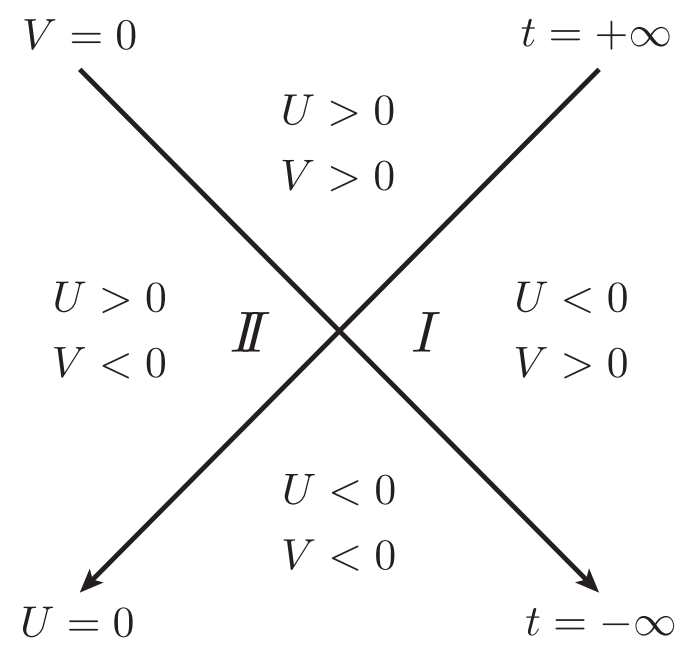

FIG. 2: The Kruskal coordinates. The right quadrant corresponds to the field variable labeled by + and the left quadrant corresponds to the field variable labeled by - .

\section{B. The semi-holographic approach for influence functional}

As in [27], the classical gravity action in (19) is the linearized Nambu-Goto action for the probed string in the background (20), and is given by

$$
S_{N G} \approx-\frac{1}{4 \pi \alpha^{\prime}} \int d r d t\left(r^{z+3} f(r) \partial_{r} X^{I} \partial_{r} X^{I I}-\frac{\partial_{t} X^{I} \partial_{t} X^{I}}{f(r) r^{z-1}}\right)
$$

where $X^{I}(t, r)$ is the linearized string perturbations in the static gauge. Since the motion of string in different directions decoupled, without losing generality we consider its motion along one of the directions denoted by $X(t, r)$. The equation of motion of the string in the frequency space, $X(t, r)=X_{\omega}(r) e^{-i \omega t}$, is given by

$$
\frac{\partial}{\partial r}\left[r^{z+3} f(r) \frac{\partial}{\partial r} X_{\omega}(r)\right]+\frac{\omega^{2}}{r^{z-1} f(r)} X_{\omega}(r)=0 .
$$

Near the horizon, the solution behaves like $X_{\omega}(r) \propto e^{ \pm i \omega r_{*}}$. Thus two linearly independent solutions, $\mathcal{X}_{\omega}(r)$ and $\mathcal{X}_{\omega}^{*}(r)$ can be found with the properties $\mathcal{X}_{\omega}(r)_{r \rightarrow r_{h}}^{\propto} e^{+i \omega r_{*}}$ and $\mathcal{X}_{\omega}^{*}(r) \underset{r \rightarrow r_{h}}{\propto} e^{-i \omega r_{*}}$. We then choose a normalization so that $\mathcal{X}_{\omega}\left(r_{b}\right)=1$. As in [39], now the general solution in the extended black hole can be parametrized as

$$
\begin{aligned}
& Q^{+}\left(\omega, r_{1}\right)=a(\omega) \mathcal{X}_{\omega}\left(r_{1}\right)+b(\omega) \mathcal{X}_{\omega}^{*}\left(r_{1}\right) \\
& Q^{-}\left(\omega, r_{2}\right)=a(\omega) \alpha_{\omega} \mathcal{X}_{\omega}\left(r_{2}\right)+b(\omega) \beta_{\omega} \mathcal{X}_{\omega}^{*}\left(r_{2}\right)
\end{aligned}
$$


Four boundary conditions are needed to fix all the parameters. Two of them are imposed at $r=r_{b}$

$$
Q^{+}\left(\omega, r_{b}\right)=q^{+}(\omega), Q^{-}\left(\omega, r_{b}\right)=q^{-}(\omega)
$$

which are interpreted as the boundary sources and identified as the position of the Brownian particle. Thus we find

$$
\begin{aligned}
& a(\omega)=\frac{1}{\alpha_{\omega}-\beta_{\omega}} q^{-}(\omega)-\frac{\beta_{\omega}}{\alpha_{\omega}-\beta_{\omega}} q^{+}(\omega), \\
& b(\omega)=\frac{\alpha_{\omega}}{\alpha_{\omega}-\beta_{\omega}} q^{+}(\omega)-\frac{1}{\alpha_{\omega}-\beta_{\omega}} q^{-}(\omega),
\end{aligned}
$$

with $\alpha_{\omega}$ and $\beta_{\omega}$ undetermined. This general solution is then plugged into the classical action in (26), resulting in a boundary term at $r=r_{b}$ as

$$
\begin{aligned}
S_{N G}=-\frac{r_{b}^{z+3}}{4 \pi \alpha^{\prime}} \int \frac{d \omega}{2 \pi}\left[Q^{+}\left(-\omega, r_{b}\right) \partial_{r} Q^{+}\left(\omega, r_{b}\right)-Q^{-}\left(-\omega, r_{b}\right) \partial_{r} Q^{-}\left(\omega, r_{b}\right)\right] \\
=-\frac{1}{2} \int \frac{d \omega}{2 \pi}\left\{q^{+}(-\omega)\left[A_{\omega} \operatorname{Re} G_{R}(\omega)+i B_{\omega} \operatorname{Im} G_{R}(\omega)\right] q^{+}(\omega)\right. \\
+q^{-}(-\omega)\left[C_{\omega} \operatorname{Re} G_{R}(\omega)+i D_{\omega} \operatorname{Im} G_{R}(\omega)\right] q^{-}(\omega) \\
-q^{+}(-\omega)\left[E_{\omega} \operatorname{Re} G_{R}(\omega)+i F_{\omega} \operatorname{Im} G_{R}(\omega)\right] q^{-}(\omega) \\
\left.-q^{-}(-\omega)\left[G_{\omega} \operatorname{Re} G_{R}(\omega)+i H_{\omega} \operatorname{Im} G_{R}(\omega)\right] q^{+}(\omega)\right\}
\end{aligned}
$$

where we have defined $G_{R}(\omega)=\frac{r_{b}^{z+3}}{2 \pi \alpha^{\prime}} \mathcal{X}_{-\omega}\left(r_{b}\right) \partial_{r} \mathcal{X}_{\omega}\left(r_{b}\right)$, and this coincides with the retarded Green function constructed by the prescription in [39]. The forms of the parameters $A_{\omega}, B_{\omega}$, ..., $H_{\omega}$ in term of $\alpha_{\omega}$ and $\beta_{\omega}$ are given in Appendix A. We can identify the action in (31) as the influence functional $\mathcal{F}\left(q^{+}, q^{-}\right)$with $q^{+}$and $q^{-}$being as sources in two respective branches of the closed-time-path contour. The field correlators are obtained by taking functional derivative

$$
G^{a b}(\omega)=\frac{\delta}{\delta q^{a}} \frac{\delta}{\delta q^{b}} S_{N G}\left[q^{+}, q^{-}\right]
$$

where $a$ and $b$ label the branches \pm .

The idea now is to propose a prescription to explicitly write down the holographic influence functional (31), that is, to determine $\alpha_{\omega}$ and $\beta_{\omega}$. The rationale of the following prescription is directly using basic properties of the influence functional to set up the dictionary. For the thermal states of the bosonic field the influence functional obeys the unitarity 
and periodicity conditions as in Eqs. (14) and (18). We first impose the unitarity condition encoded in (18) and find

$$
\begin{aligned}
& A_{\omega}+C_{\omega}+E_{\omega}+F_{\omega}=0, \\
& B_{\omega}+E_{\omega}+F_{\omega}+G_{\omega}=0 .
\end{aligned}
$$

The solution is either $\alpha_{\omega}=1, \beta_{-\omega}=\beta_{\omega}^{-1}$ or $\beta_{\omega}=1, \alpha_{-\omega}=\alpha_{\omega}^{-1}$ where both $\alpha_{\omega}$ and $\beta_{\omega}$ are real functions of $\omega$ (see Appendix A). These two solutions will give the same influence functional as we will see later. Let us choose the first one and we have

$$
\begin{aligned}
G^{++}(\omega) & =\operatorname{Re} G_{R}(\omega)+\frac{\beta_{\omega}+1}{\beta_{\omega}-1} i \operatorname{Im} G_{R}(\omega), \\
G^{--}(\omega) & =-\operatorname{Re} G_{R}(\omega)+\frac{\beta_{\omega}+1}{\beta_{\omega}-1} i \operatorname{Im} G_{R}(\omega), \\
G^{+-}(\omega) & =\frac{2 \beta_{\omega}}{\beta_{\omega}-1} i \operatorname{Im} G_{R}(\omega), \\
G^{-+}(\omega) & =\frac{2}{\beta_{\omega}-1} i \operatorname{Im} G_{R}(\omega) .
\end{aligned}
$$

Here $\beta_{\omega}$ remains undetermined. Finally since the periodicity condition in (14) requires

$$
\frac{G^{+-}(\omega)}{G^{-+}(\omega)}=e^{-\frac{\omega}{T}}
$$

we use it to fix $\beta_{\omega}$ and find $\beta_{\omega}=e^{-\frac{\omega}{T}}$. As for the general contour [51] with a finite $\sigma$ seen in Fig. 1, the unitarity condition becomes

$$
G^{++}(\omega)-e^{\omega \sigma} G^{+-}(\omega)-e^{-\omega \sigma} G^{-+}(\omega)+G^{--}(\omega)=0
$$

Accordingly the periodicity condition for the thermal bath is modified to the form

$$
\frac{G^{+-}(\omega)}{G^{-+}(\omega)}=e^{-\frac{\omega}{T}+2 \sigma \omega}
$$

Putting all together, we will arrive at $\alpha_{\omega}=e^{-\omega \sigma}$ and $\beta_{\omega}=e^{-\omega \sigma} e^{\frac{\omega}{T}}$. The corresponding nonequilibrium correlators for general $\sigma$ is obtained as

$$
\begin{aligned}
& G^{++}(\omega)=\operatorname{Re} G_{R}(\omega)+(1+2 n) i \operatorname{Im} G_{R}(\omega), \\
& G^{--}(\omega)=-\operatorname{Re} G_{R}(\omega)+(1+2 n) i \operatorname{Im} G_{R}(\omega), \\
& G^{+-}(\omega)=-2 n e^{\omega \sigma} i \operatorname{Im} G_{R}(\omega), \\
& G^{-+}(\omega)=-2(1+n) e^{-\omega \sigma} i \operatorname{Im} G_{R}(\omega),
\end{aligned}
$$


where $n=\left(e^{\frac{\omega}{T}}-1\right)^{-1}$ is the occupation number. Especially, in the context of quantum decoherence, two particular combinations of these correlators are useful,

$$
\begin{aligned}
& G_{R}\left(t-t^{\prime}\right)=\left\{G^{++}\left(t-t^{\prime}\right)-G^{+-}\left(t-t^{\prime}\right)\right\}, \\
& G_{H}\left(t-t^{\prime}\right)=\frac{i}{4}\left\{G^{++}\left(t-t^{\prime}\right)+G^{+-}\left(t-t^{\prime}\right)+G^{++}\left(t-t^{\prime}\right)+G^{+-}\left(t-t^{\prime}\right)\right\} .
\end{aligned}
$$

Therefore the holographic influence functional is fully constructed by the prescription we propose here.

\section{QUANTUM DECOHERENCE INDUCED BY STRONGLY COUPLED FIELDS}

We now apply the obtained holographic influence functional to the quantum decoherence problem, in particular, for the Brownian particle moving in the strongly coupled quantum critical theories. For the linear coupling between the system and environment, the reduced density matrix and the influence functional are found in (8) and (11) respectively. We then follow the approach in [45] to set up our interference experiment. To proceed, we express the influence functional in a form of its phase and modulus by:

$$
\mathcal{F}\left[q^{+}, q^{-}\right]=\exp \left\{\mathcal{W}\left[q^{+}, q^{-}\right]+i \Phi\left[q^{+}, q^{-}\right]\right\}
$$

where the real functionals $\mathcal{W}$ and $\Phi$ are

$$
\begin{gathered}
\Phi\left[q^{+}, q^{-}\right]=\frac{1}{2} \int d t \int d t^{\prime}\left[q^{+}(t)-q^{-}\left(t^{\prime}\right)\right] G_{R}\left(t-t^{\prime}\right)\left[q^{+}(t)+q^{-}\left(t^{\prime}\right)\right] \\
\mathcal{W}\left[q^{+}, q^{-}\right]=-\frac{1}{2} \int d t \int d t^{\prime}\left[q^{+}(t)-q^{-}\left(t^{\prime}\right)\right] G_{H}\left(t-t^{\prime}\right)\left[q^{+}(t)-q^{-}\left(t^{\prime}\right)\right] .
\end{gathered}
$$

The retarded Green's function and the Hadamard function of the environmental field are respectively defined by

$$
\begin{aligned}
G_{R}\left(t-t^{\prime}\right) & =-i \theta\left(t-t^{\prime}\right)\left\langle\left[F(t), F\left(t^{\prime}\right)\right]\right\rangle, \\
G_{H}\left(t-t^{\prime}\right) & =\frac{1}{2}\left\langle\left\{F(t), F\left(t^{\prime}\right)\right\}\right\rangle .
\end{aligned}
$$

For a given initial state for the particle, the reduced density matrix at time $t_{f}$ can be obtained from (8) when the path integration over $q^{ \pm}$in (9) is carried out. Expressed explicitly in 
terms of the phase and modulus of the influence functional, the reduced density matrix now becomes

$$
\begin{aligned}
\rho_{r}\left(q_{f}, \tilde{q}_{f}, t_{f}\right)=\int d q_{1} d q_{2} & {\left[\int_{q_{1}}^{q_{f}} \mathcal{D} q^{+} \int_{q_{2}}^{\tilde{q}_{f}} \mathcal{D} q^{-} \exp \left\{i \int_{t_{i}}^{t_{f}} d t\left(L_{q}\left[q^{+}\right]-L_{q}\left[q^{-}\right]\right)\right\}\right.} \\
& \left.\times \exp \left\{\mathcal{W}\left[q^{+}, q^{-}\right]\right\} \exp \left\{i \Phi\left[q^{+}, q^{-}\right]\right\}\right] \rho_{q}\left(q_{1}, q_{2}, t_{i}\right) .
\end{aligned}
$$

Suppose that the initial state $\left|\Psi\left(t_{i}\right)\right\rangle$ of the particle is a coherent superposition of two localized states, which will travel along the two non-overlapping paths $C_{1}$ and $C_{2}$. If both states leave the same spatial point at the moment $t_{i}$,

$$
\left|\Psi\left(t_{i}\right)\right\rangle=\left|\psi_{1}\left(t_{i}\right)\right\rangle+\left|\psi_{2}\left(t_{i}\right)\right\rangle
$$

then the initial density matrix of the state can be written as

$$
\rho_{q}\left(t_{i}\right)=\left|\Psi\left(t_{i}\right)\right\rangle\left\langle\Psi\left(t_{i}\right)\right|=\rho_{11}\left(t_{i}\right)+\rho_{22}\left(t_{i}\right)+\rho_{21}\left(t_{i}\right)+\rho_{12}\left(t_{i}\right),
$$

where $\rho_{m n}\left(t_{i}\right)=\left|\psi_{m}\left(t_{i}\right)\right\rangle\left\langle\psi_{n}\left(t_{i}\right)\right|$. The term $\rho_{21}+\rho_{12}$ accounts for quantum interference, because when the density matrix is realized in the coordinate basis, we have

$$
\left\langle q_{i}\left|\rho_{q}\left(t_{i}\right)\right| q_{i}\right\rangle=\left|\psi_{1}\left(q_{i}, t_{i}\right)\right|^{2}+\left|\psi_{2}\left(q_{i}, t_{i}\right)\right|^{2}+2 \operatorname{Re}\left\{\psi_{2}^{*}\left(q_{i}, t_{i}\right) \psi_{1}\left(q_{i}, t_{i}\right)\right\}
$$

which expresses the probability of finding the particle at $\left(t_{i}, q_{i}\right)$ in the superposed state.

Next let these two localized states $\left|\psi_{m}\right\rangle$ move along the respective path $C_{m}$, and then they are recombined at the location $q_{f}$ at later time $t_{f}$. The corresponding spacetime paths are drawn in Fig. 3. The interference pattern of the superposed state at $t_{f}$ is given by the diagonal elements of the reduced density matrix $\left\langle q_{f}\left|\rho_{r}\left(t_{f}\right)\right| q_{f}\right\rangle=\rho_{r}\left(q_{f}, q_{f}, t_{f}\right)$. The mean trajectory of the wave packets $\left|\psi_{m}\right\rangle$ follows the classical path constrained by an appropriate external potential. We assume that the finite spread of the wave packet of the state can be legitimately neglected as long as the de Broglie wavelength, $\lambda_{\mathrm{dB}}$ is much shorter than the characteristic length scale associated with the accuracy of the measurement $\ell_{0}$, about the same order as travel distance for the states to recombine. Thus, when $\ell_{0} \gg \lambda_{\mathrm{dB}}$, the wave packet can be seen to remain quite sharply peaked in its position and momentum, thus we may ignore the effect of wavefunction spreading. As such, the leading effect of the decoherence can be obtained by evaluating the propagating function (9) along a prescribed classical path. The diagonal components of the reduced density matrix $\rho_{r}\left(q_{f}, q_{f}, t_{f}\right)$ now 


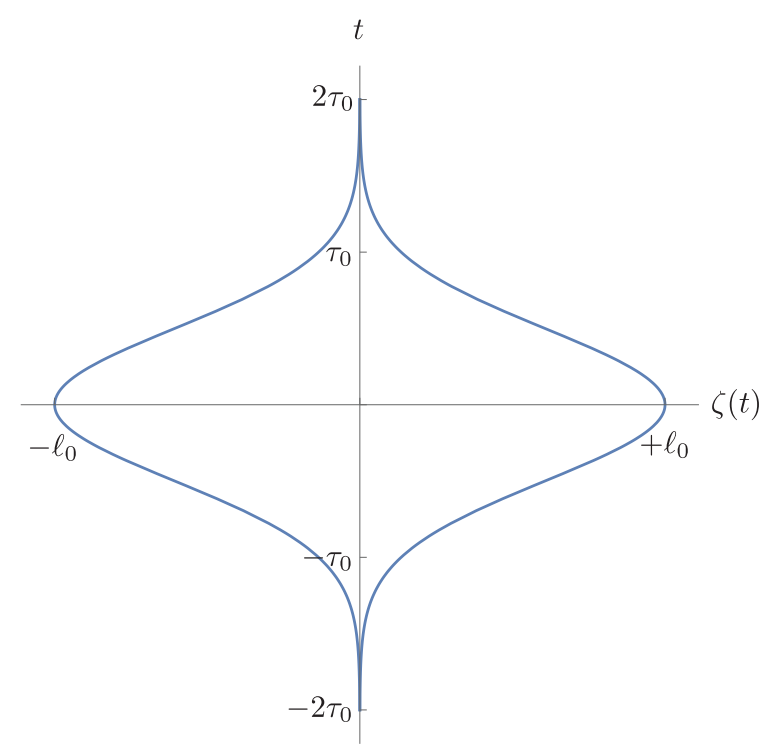

FIG. 3: The spacetime paths of the wavepackets for an interference experiment.

becomes

$\rho_{r}\left(q_{f}, q_{f}, t_{f}\right)=\left|\psi_{1}\left(q_{f}, t_{f}\right)\right|^{2}+\left|\psi_{2}\left(q_{f}, t_{f}\right)\right|^{2}+2 e^{\mathcal{W}\left[\bar{q}^{+}, \bar{q}^{-}\right]} \operatorname{Re}\left\{e^{i \Phi\left[\bar{q}^{+}, \bar{q}^{-}\right]} \psi_{1}\left(q_{f}, t_{f}\right) \psi_{2}^{*}\left(q_{f}, t_{f}\right)\right\}$,

where the $\mathcal{W}$ and $\Phi$ functionals are evaluated along the classical trajectories, $C_{1}=\bar{q}^{+}$and $C_{2}=\bar{q}^{-}$.

The decoherence functional $\mathcal{W}$, determined by the expectation value of the anticommutator of environmental field operators, reveals decoherence between particle states, while its phase functional $\Phi$, related to the expectation value of the commutator of field operators, results in an overall phase shift in the interference pattern. Both effects arise from the interaction with quantum fields. It can be seen that the effects of quantum decoherence and the phase shift are likely to be related by the fluctuation-dissipation relation

$$
G_{H}(\omega)=-\operatorname{coth}\left[\frac{\omega}{2 T}\right] \operatorname{Im} G_{R}(\omega) .
$$

At zero temperature $T \rightarrow 0$, the relation reduces to

$$
G_{H}(\omega)=-\operatorname{sgn}(\omega) \operatorname{Im} G_{R}(\omega)
$$

Here we consider the prescribed trajectories $\bar{q}^{ \pm}= \pm \zeta(t)$ of the localized states. The path function $\zeta(t)$ is required to be sufficiently smooth and is chosen to take the form,

$$
\zeta(t)=\ell_{0} e^{-\frac{t^{2}}{\tau_{0}^{2}}}
$$


where $2 \ell_{0}$ is the effective path separation and $2 \tau_{0}$ is the effective flight time as in [45] (See Fig. 3). We then have the decoherence functional from (43)

$$
\mathcal{W}=-\int \frac{d \omega}{2 \pi} G_{H}(\omega) \int d t\left|\zeta(t) e^{-i \omega t}\right|^{2}<0 .
$$

Since these two prescribed classical trajectories are symmetric with respect to the initial position, it implies $q^{+}(t)+q^{-}(t)=0$ and in turns we will not see any phase shift, that is, $\Phi=0$ from (42). We now use the holographic influence functional to evaluate $\mathcal{W}$.

\section{A. Holography at zero temperature}

In the general Liftshitz black hole background, the analytic expression for $G_{R}(\omega)=$ $\frac{r_{b}^{z+3}}{2 \pi \alpha^{\prime}} X_{-\omega}\left(r_{b}\right) \partial_{r} X_{\omega}\left(r_{b}\right)$ cannot be obtained except in the zero temperature limit where it is given by (see [27] and [20])

$$
G_{R}(\omega)=\frac{\omega r_{b}^{2}}{2 \pi \alpha^{\prime}} \frac{H_{\frac{1}{z}-\frac{1}{2}}^{(1)}\left(\frac{\omega}{z r_{b}^{z}}\right)}{H_{\frac{1}{z}+\frac{1}{2}}^{(1)}\left(\frac{\omega}{z r_{b}^{z}}\right)}
$$

Here $H_{\nu}^{(1)}(z)$ is Hankel's function and it takes values in the principle branch $-\pi<\arg (z)<\pi$ in order for $G_{R}(\omega)$ to be analytic on the upper half of $\omega$-plane. The Hadamard function can be found from the retarded Green's function by means of the fluctuation-dissipation relation. Its leading contribution in the small frequency limit is

$$
G_{H}(\omega) \simeq \frac{1}{2 \pi \alpha^{\prime}} \frac{\cos \left(\frac{\pi}{z}\right)}{(2 z)^{\frac{z}{2}}} \frac{\Gamma\left(\frac{1}{2}-\frac{1}{z}\right)}{\Gamma\left(\frac{1}{2}+\frac{1}{z}\right)} \operatorname{sgn}(\omega) \omega^{1+\frac{2}{z}}+\mathcal{O}\left(\omega^{2} / r_{b}^{2 z}\right)
$$

For the general $z$ the leading effect to the decoherence functional is found to be

$$
\mathcal{W} \simeq-\frac{2}{\pi \alpha^{\prime}} \frac{\cos \left(\frac{\pi}{z}\right)}{(z)^{\frac{z}{2}}} \frac{\Gamma\left(1+\frac{1}{z}\right) \Gamma\left(\frac{1}{2}-\frac{1}{z}\right)}{\Gamma\left(\frac{1}{2}+\frac{1}{z}\right)} \frac{\ell_{0}^{2}}{\tau_{0}^{\frac{2}{z}}}+\mathcal{O}\left(1 / r_{b}^{2 z} \tau_{0}^{2}\right)
$$

The $\mathcal{W}$ functional is smooth cross $z=2$. In [27] the holographic method is used to study fluctuations and dissipation of an $n$-dimensional moving-mirror coupled to quantum critical theories. The effects of the environment on the system are classified according to the lowfrequency behavior of the the friction term $\gamma \omega^{k+1}$, as subohmic, ohmic, and supraohmic for $k<0, k=0$ and $k>0$ respectively. We then found that the low-frequency expansion of the damping term by the holographic approach gives $k=(n+2) / z$. For a point particle, which corresponds to the $n=0$ case, the dissipative dynamics due to these quantum critical 
theories with $z$ not much larger than 2 is supraohmic. However, when $z \gg 2$, the dissipative dynamics then demonstrates the ohmic behavior. Thus, for a fixed travel time $\tau_{0}$, the decoherence effect decreases as $z$ increases toward the ohmic situation. This is one of our main results.

In particular, for $z=1$ (the relativistic environmental field), the decoherence function reduces to

$$
\mathcal{W} \simeq-\frac{4}{\pi \alpha^{\prime}} \frac{\ell_{0}^{2}}{\tau_{0}^{2}}+\mathcal{O}\left(1 / r_{b}^{2} \tau_{0}^{2}\right)
$$

with velocity squared dependence. This is the same as the result in [52], but the proportionality constant are different between the strongly coupled environment and the free field background, as expected. Notice that it will always show enhancement in the strong field theory with coupling $\lambda>>1$ through the relation $\lambda=L^{4} / \alpha^{2}$, as the consequence of AdS/CFT correspondence, where $L$ is the curvature radius in the Lifshitz background.

\section{B. Holography in high temperature}

In the finite temperature case, the corresponding retarded Green's function (the response function) has been studied in [20, 27], and in the low frequency and high temperature approximation $\left(\frac{r_{h}}{r_{b}}>>\frac{\omega}{r_{b}^{z}}\right)$, this is given by

$$
G_{R}^{(T)}(\omega)=-\gamma_{T}(z) i \omega+m_{T}(z)(i \omega)^{2}+\mathcal{O}\left(\omega^{3}\right),
$$

where

$$
m_{T}(z)=\frac{1}{2 \pi \alpha^{\prime}} \frac{1}{r_{b}^{z-2}}\left(\frac{r_{h}}{r_{b}}\right)^{4}\left[(2+z)-\kappa r_{b}^{z+2}\right], \quad \gamma_{T}(z)=\frac{1}{2 \pi \alpha^{\prime}} r_{h}^{2} .
$$

The parameter $\kappa$ is the integration constant depends on the detail of black hole geometry. The inertial mass $m_{T}$ and the damping coefficient $\gamma_{T}$ have the temperature dependence through the black hole temperature (21). Since the damping term has linear $\omega$ dependence, the stochastic dynamics of the particle in the thermal environment will be ohmic. By the fluctuation-dissipation relation (51) the Hadamard function at high $T$ is obtained as

$$
G_{H}^{(T)}(\omega) \simeq 2 T \gamma_{T}(z)=\frac{2 T}{2 \pi \alpha^{\prime}}\left(\frac{4 \pi T}{z+3}\right)^{\frac{2}{z}}
$$

This gives

$$
\mathcal{W}_{T} \simeq-\frac{16 \pi \ell_{0}^{2} \tau_{0}}{\alpha^{\prime}} \sqrt{\frac{\pi}{2}} T\left(\frac{4 \pi T}{z+3}\right)^{\frac{2}{z}}
$$


Compared with the case of the free environment field, the same fluctuation-dissipation relation at finite temperature (51) is found in [46, 53]. However, a distinctive feature of the free field case is that the linear coupling between the particle and the environment field leads to a damping coefficient $\gamma$ that does not depend on the state of the environment and thus is independent of temperature [53]. Thus, in the free field case, the $\mathcal{W}$ functional reveals linear dependence in temperature. This is in dramatic difference from the strongly coupled environment where Eq. (60) shows the temperature dependence of $\gamma$. The additional $T$ dependence certainly arises from the effects from strong self-interaction of the environment field, and can not be obtained by a weak coupling expansion. Therefore, the decoherence effect will be enhanced from not only the strong coupling of the environment field itself, but also the finite temperature effects. Similar to the zero temperature case, the decoherence effect also decreases as $z$ increases. In order to reduce the decoherence effects from quantum critical theories, the larger value of the dynamical exponent $z$ is suggested.

\section{CONCLUDING REMARKS}

Understanding how the environment affects the system of interest is the main concerns in nonequilibrium statistical mechanics. By tracing out the environment degrees of freedom, its effects on the system can be encoded in the influence functional. In this paper a probed particle in the strongly coupled quantum critical theories with general dynamical scaling $z$ is considered. The holographic setup is a probed string in the classical Lifshitz geometry. We

first adapt the approach in [39] to construct the holographic influence functional. However it is modified based on the field-theoretic consideration. We then apply the holographic influence functional to study the decoherence of the particle induced by strongly coupled quantum critical theories. We find that the decoherence effect gets enhanced as the coupling of the environmental field becomes strong. Nevertheless, when the dynamical scaling $z$ increases, the quantum critical theories transit from a supraohmic to an ohmic environment, and the decoherence effect becomes less significant. Additionally the decoherence effect will be enhanced not only by its strong coupling constant but also from the finite temperature effects that certainly can not be achieved by a weak coupling expansion. Similar to the zero temperature case, the larger value of the dynamical exponent $z$ is suggested to reduce the decoherence effect at finite temperature from quantum critical theories. 
Furthermore, this revised prescription may pave a way for extending the holographic method of influence functional to more general states such as squeezed vacuum state [49] so as to consider the potential "subvacuum" phenomena [47]. Manipulating the quantum field may give rise to suppression of its vacuum uncertainties. One of the known examples is the squeezed vacuum state, from which the decoherence of the particle can be suppressed below

the level due to the pure vacuum state, leading to the so-called subvacuum phenomena [44]. It is also known that subvacuum phenomena can not last for an arbitrarily long period of time. However, so far all the previous studies consider only the free field, so its effect on the system is expected to be weak. Thus, our future work will explore this phenomenon in a strongly coupled field theory in the hope that it will be significantly amplified so as to be more observable in the experiment realization.

\section{Acknowledgments}

This work was supported in part by the National Science Council, Taiwan.

\section{Appendix A: coefficients for the holographic influence functional}

In this appendix, we write down the Schwinger-Keldysh correlators in term of two unknown parameters $\alpha_{\omega}$ and $\beta_{\omega}$. We will determine one of them using the unitarity requirement 
in the zero $\sigma$ case. In the section III-B, the functions $A_{\omega}, B_{\omega}, \ldots, H_{\omega}$ are

$$
\begin{aligned}
A_{\omega}= & \frac{\left(1-\alpha_{\omega} \alpha_{-\omega}\right) \beta_{\omega} \beta_{-\omega}-\left(1-\alpha_{\omega} \beta_{-\omega}\right) \beta_{\omega} \alpha_{-\omega}-\left(1-\beta_{\omega} \alpha_{-\omega}\right) \alpha_{\omega} \beta_{-\omega}+\left(1-\beta_{\omega} \beta_{-\omega}\right) \alpha_{\omega} \alpha_{-\omega}}{\left(\alpha_{\omega}-\beta_{\omega}\right)\left(\alpha_{-\omega}-\beta_{-\omega}\right)}, \\
B_{\omega}= & \frac{\left(1-\alpha_{\omega} \alpha_{-\omega}\right) \beta_{\omega} \beta_{-\omega}-\left(1-\alpha_{\omega} \beta_{-\omega}\right) \beta_{\omega} \alpha_{-\omega}+\left(1-\beta_{\omega} \alpha_{-\omega}\right) \alpha_{\omega} \beta_{-\omega}-\left(1-\beta_{\omega} \beta_{-\omega}\right) \alpha_{\omega} \alpha_{-\omega}}{\left(\alpha_{\omega}-\beta_{\omega}\right)\left(\alpha_{-\omega}-\beta_{-\omega}\right)}, \\
C_{\omega}= & \frac{-\left(1-\alpha_{\omega} \alpha_{-\omega}\right) \beta_{\omega}+\left(1-\alpha_{\omega} \beta_{-\omega}\right) \beta_{\omega}+\left(1-\beta_{\omega} \alpha_{-\omega}\right) \alpha_{\omega}-\left(1-\beta_{\omega} \beta_{-\omega}\right) \alpha_{\omega}}{\left(\alpha_{\omega}-\beta_{\omega}\right)\left(\alpha_{-\omega}-\beta_{-\omega}\right)}, \\
D_{\omega}= & \frac{-\left(1-\alpha_{\omega} \alpha_{-\omega}\right) \beta_{\omega}+\left(1-\alpha_{\omega} \beta_{-\omega}\right) \beta_{\omega}-\left(1-\beta_{\omega} \alpha_{-\omega}\right) \alpha_{\omega}+\left(1-\beta_{\omega} \beta_{-\omega}\right) \alpha_{\omega}}{\left(\alpha_{\omega}-\beta_{\omega}\right)\left(\alpha_{-\omega}-\beta_{-\omega}\right)}, \\
E_{\omega}= & \frac{-\left(1-\alpha_{\omega} \alpha_{-\omega}\right) \beta_{-\omega}+\left(1-\alpha_{\omega} \beta_{-\omega}\right) \alpha_{-\omega}+\left(1-\beta_{\omega} \alpha_{-\omega}\right) \beta_{-\omega}-\left(1-\beta_{\omega} \beta_{-\omega}\right) \alpha_{-\omega}}{\left(\alpha_{\omega}-\beta_{\omega}\right)\left(\alpha_{-\omega}-\beta_{-\omega}\right)} \\
F_{\omega}= & \frac{-\left(1-\alpha_{\omega} \alpha_{-\omega}\right) \beta_{-\omega}+\left(1-\alpha_{\omega} \beta_{-\omega}\right) \alpha_{-\omega}-\left(1-\beta_{\omega} \alpha_{-\omega}\right) \beta_{-\omega}+\left(1-\beta_{\omega} \beta_{-\omega}\right) \alpha_{-\omega}}{\left(\alpha_{\omega}-\beta_{\omega}\right)\left(\alpha_{-\omega}-\beta_{-\omega}\right)}, \\
G_{\omega}= & \frac{\left(1-\alpha_{\omega} \alpha_{-\omega}\right)-\left(1-\alpha_{\omega} \beta_{-\omega}\right) \alpha_{-\omega}-\left(1-\beta_{\omega} \alpha_{-\omega}\right)+\left(1-\beta_{\omega} \beta_{-\omega}\right)}{\left(\alpha_{\omega}-\beta_{\omega}\right)\left(\alpha_{-\omega}-\beta_{-\omega}\right)}, \\
H_{\omega}= & \frac{\left(1-\alpha_{\omega} \alpha_{-\omega}\right)-\left(1-\alpha_{\omega} \beta_{-\omega}\right) \alpha_{-\omega}+\left(1-\beta_{\omega} \alpha_{-\omega}\right)-\left(1-\beta_{\omega} \beta_{-\omega}\right)}{\left(\alpha_{\omega}-\beta_{\omega}\right)\left(\alpha_{-\omega}-\beta_{-\omega}\right)} .
\end{aligned}
$$

By (32) we can write the Schwinger-Keldysh correlators as

$$
\begin{aligned}
& G^{++}(\omega)=A(\omega) \operatorname{Re} G_{R}(\omega)+i B(\omega) \operatorname{Im} G_{R}(\omega), \\
& G^{--}(\omega)=C(\omega) \operatorname{Re} G_{R}(\omega)+i D(\omega) \operatorname{Im} G_{R}(\omega), \\
& G^{+-}(\omega)=-E(\omega) \operatorname{Re} G_{R}(\omega)-i F(\omega) \operatorname{Im} G_{R}(\omega), \\
& G^{-+}(\omega)=-G(\omega) \operatorname{Re} G_{R}(\omega)-i H(\omega) \operatorname{Im} G_{R}(\omega) .
\end{aligned}
$$

The unitarity condition for zero $\sigma$ says that

$$
G^{++}(\omega)-G^{+-}(\omega)-G^{-+}(\omega)+G^{--}(\omega)=0 .
$$

In the following we will assume that $\alpha_{\omega}$ and $\beta_{\omega}$ are real. From the unitarity and the periodicity conditions we will find that this is the consistent assumption. Thus the real part of this equation gives

$$
\begin{aligned}
A_{\omega}+C_{\omega}+E_{\omega}+F_{\omega}= & \left(1-\alpha_{\omega} \alpha_{-\omega}\right)\left(\beta_{\omega} \beta_{-\omega}-\beta_{\omega}-\beta_{-\omega}+1\right) \\
& +\left(1-\alpha_{\omega} \beta_{-\omega}\right)\left(-\beta_{\omega} \alpha_{-\omega}+\beta_{\omega}+\alpha_{-\omega}-1\right) \\
& +\left(1-\beta_{\omega} \alpha_{-\omega}\right)\left(-\alpha_{\omega} \beta_{-\omega}+\alpha_{\omega}+\beta_{-\omega}-1\right) \\
& +\left(1-\beta_{\omega} \beta_{-\omega}\right)\left(\alpha_{\omega} \alpha_{-\omega}-\alpha_{\omega}-\alpha_{-\omega}+1\right) \\
= & 0 .
\end{aligned}
$$


One solution to this equation is $\alpha_{-\omega}=\alpha_{\omega}^{-1}$ and $\beta_{-\omega}=\beta_{\omega}^{-1}$. The imaginary part of (A3), $A_{\omega}+C_{\omega}+E_{\omega}+F_{\omega}=0$, then yields to

$$
\left(1-\frac{\alpha_{\omega}}{\beta_{\omega}}\right)\left(-\frac{\beta_{\omega}}{\alpha_{\omega}}+\beta_{\omega}+\frac{1}{\alpha_{\omega}}-1\right)-\left(1-\frac{\beta_{\omega}}{\alpha_{\omega}}\right)\left(-\frac{\alpha_{\omega}}{\beta_{\omega}}+\alpha_{\omega}+\frac{1}{\beta_{\omega}}-1\right)=0 .
$$

Either $\alpha_{\omega}=1$ or $\beta_{\omega}=1$ is a solution.

\section{Appendix B: using the boundary condition at the horizon}

In this appendix we determine the solution of the equation (27) by imposing the boundary condition at the horizon, proposed in [39]. As discussed in the main text, we have the two linearly-independent solutions. One is $e^{-i \omega t} \mathcal{X}_{\omega}(r) \underset{r \rightarrow r_{h}}{\longrightarrow} e^{-i \omega\left(t+r_{*}\right)}=e^{-i \frac{\omega}{2 \pi T} \ln V}$ and the other is $e^{-i \omega t} \mathcal{X}_{\omega}^{*}(r) \underset{r \rightarrow r_{h}}{\longrightarrow} e^{i \omega\left(-t+r_{*}\right)}=e^{i \frac{\omega}{2 \pi T} \ln (-U)}$, where $U$ and $V$ are Kruskal coordinates defined in (24)

The general solution in the extended black hole background is

$$
\begin{aligned}
& Q^{+}\left(\omega, r_{1}\right)=a(\omega) \mathcal{X}_{\omega}\left(r_{1}\right)+b(\omega) \mathcal{X}_{\omega}^{*}\left(r_{1}\right) \\
& Q^{-}\left(\omega, r_{2}\right)=c(\omega) \mathcal{X}_{\omega}\left(r_{2}\right)+d(\omega) \mathcal{X}_{\omega}^{*}\left(r_{2}\right)
\end{aligned}
$$

We impose two boundary conditions at $r_{1}=r_{b}$ and $r_{2}=r_{b}$ as in (29). The other two boundary conditions are imposed on the horizon. As proposed in [39], in order to obtain the right correlators, they required the modes with positive (negative) frequency are purely incoming (outgoing). (By definition, in the region $\mathbb{I}$, the outgoing wave is coming in to the horizon). The modes that satisfy these boundary conditions are

$$
e^{-i \omega t} \mathcal{X}_{\omega}\left(r_{1}\right)+e^{-\frac{\omega}{2 T}} e^{-i \omega t} \mathcal{X}_{\omega}\left(r_{2}\right) \underset{r \rightarrow r_{h}}{\longrightarrow} e^{-\frac{i \omega}{2 \pi T} \ln (V)},
$$

which is incoming with positive frequency (analytic in lower half $V$-plane), and

$$
e^{-i \omega t} \mathcal{X}_{\omega}^{*}\left(r_{1}\right)+e^{\frac{\omega}{2 T}} e^{-i \omega t} \mathcal{X}_{\omega}^{*}\left(r_{2}\right) \underset{r \rightarrow r_{h}}{\longrightarrow} e^{\frac{i \omega}{2 \pi T} \ln (-U)}
$$

which is outgoing with negative frequency (analytic in upper half $U$-plane). This amounts to requiring that the solutions in the region $I I$ is a continuation of the ones in the region $I$,

$$
\begin{aligned}
& Q^{+}\left(\omega, r_{1}\right)=a(\omega) \mathcal{X}_{\omega}\left(r_{1}\right)+b(\omega) \mathcal{X}_{\omega}^{*}\left(r_{1}\right) \\
& Q^{-}\left(\omega, r_{2}\right)=a(\omega) e^{-\frac{\omega}{2 T}} \mathcal{X}_{\omega}\left(r_{2}\right)+b(\omega) e^{\frac{\omega}{2 T}} \mathcal{X}_{\omega}^{*}\left(r_{2}\right)
\end{aligned}
$$


Then $a(\omega)$ and $b(\omega)$ can be determined by the boundary conditions at $r=r_{b}$. We choose a normalization so that $X_{\omega}\left(r=r_{b}\right)=1$, which gives

$$
\begin{aligned}
& a(\omega)=q^{+}(\omega)(1+n)-q^{-}(\omega) e^{\frac{\omega}{2 T}} n, \\
& b(\omega)=q^{-}(\omega) e^{\frac{\omega}{2 T}} n-q^{+}(\omega) n,
\end{aligned}
$$

where $n=\left(e^{\omega / T}-1\right)^{-1}$. Plugging the solutions (B4) into the gravity action and keeping terms to the quadratic order, we obtain

$$
\begin{aligned}
& Z\left(q^{+}, q^{-}\right)=S_{\text {gravity }} \\
&=-\frac{r_{b}^{z+3}}{4 \pi \alpha^{\prime}} \int \frac{d \omega}{2 \pi}\left(Q^{+}\left(-\omega, r_{b}\right) \partial r Q^{+}\left(\omega, r_{b}\right)-Q^{-}\left(-\omega, r_{b}\right) \partial r Q^{-}\left(\omega, r_{b}\right)\right) \\
&=-\frac{1}{2 \pi \alpha^{\prime}} \int \frac{d \omega}{2 \pi}\left\{q^{+}(-\omega)\left[i \operatorname{Re} G_{R}(\omega)-(1+2 n) \operatorname{Im} G_{R}(\omega)\right] q^{-}(\omega)\right. \\
&+q^{-}(-\omega)\left[-i \operatorname{Re} G_{R}(\omega)-(1+2 n) \operatorname{Im} G_{R}(\omega)\right] q^{-}(\omega) \\
&-q^{+}(-\omega)\left[-2 n e^{\frac{\omega}{2 T}} \operatorname{Im} G_{R}(\omega)\right] q^{-}(\omega) \\
&\left.-q^{-}(-\omega)\left[-2(1+n) e^{-\frac{\omega}{2 T}} \operatorname{Im} G_{R}(\omega)\right] q^{+}(\omega)\right\},
\end{aligned}
$$

where $G_{R}(\omega)=\mathcal{X}_{\omega}\left(r_{b}\right) \partial_{r} \mathcal{X}_{\omega}\left(r_{b}\right)$ is the retarded correlator. This prescription naturally gives the Schwinger-Keldysh correlators in the case $\sigma=\frac{1}{2 T}$ as compared with (38).

[1] J. M. Maldacena, Adv. Theor. Math. Phys. 2, 231 (1998) [Int. J. Theor. Phys. 38, 1113 (1999)]; S. S. Gubser, I. R. Klebanov and A. M. Polyakov, Phys. Lett. B 428, 105 (1998); E. Witten, Adv. Theor. Math. Phys. 2, 253 (1998).

[2] S. Hartnoll, Class.Quant.Grav. 26, 224002 (2009).

[3] M. Rangamani, Class.Quant.Grav. 26, 224003 (2009).

[4] C. P. Herzog, A. Karch, P. Kovtun, C. Kozcaz, and L. G. Yaffe, JHEP 07, 013 (2006).

[5] S. S. Gubser,Phys. Rev. D 74, 126005 (2006).

[6] J. Casalderrey-Solana and D. Teaney, Phys. Rev. D 74, 085012 (2006).

[7] D. T. Son and D. Teaney, JHEP 07, 021 (2009).

[8] S. S. Gubser, Nucl. Phys. B 790175 (2008).

[9] J. Casalderrey-Solana and D. Teaney, JHEP 04039 (2007). 
[10] E. Caceres, M. Chernicoff, A. Guijosa and J. F. Pedraza, JHEP 06, 078 (2010).

[11] G. C. Giecold, E. Iancu and A. H. Mueller, JHEP 07, 033 (2009).

[12] J. Casalderrey-Solana, K. -Y. Kim and D. Teaney, JHEP 12, 066 (2009).

[13] A. N. Atmaja, J. de Boer and M. Shigemori, Nucl. Phys. B 880, 23 (2014).

[14] S. R. Das, T. Nishioka and T. Takayanagi, JHEP 07, 071 (2010).

[15] U. Gursoy, E. Kiritsis, L. Mazzanti and F. Nitti, JHEP 12, 088 (2010).

[16] H. Ebrahim and M. Headrick, arXiv:1010.5443 [hep-th].

[17] S. Caron-Huot, P. M. Chesler and D. Teaney, Phys. Rev. D 84, 026012 (2011).

[18] E. Kiritsis, L. Mazzanti and F. Nitti, J. Phys. G 39, 054003 (2012) arXiv:1111.1008 [hep-th]].

[19] W. Fischler, J. F. Pedraza and W. Tangarife Garcia, JHEP 12, 002 (2012).

[20] D. Tong and K. Wong, Phys. Rev. Lett. 110, 061602 (2013).

[21] M. Edalati, J. F. Pedraza and W. Tangarife Garcia, Phys. Rev. D 87, 046001 (2013).

[22] A. N. Atmaja, JHEP 04, 021 (2013).

[23] J. Sadeghi, F. Pourasadollah and H. Vaez, Adv. High Energy Phys. 2014762151 (2014).

[24] A. N. Atmaja, arXiv:1308.3014 [hep-th].

[25] P. Banerjee and B. Sathiapalan, Nucl. Phys. B 88474 (2014).

[26] D. Giataganas and H. Soltanpanahi, Phys. Rev. D 89, 026011 (2014).

[27] C. -P. Yeh, J. -T. Hsiang and D. -S. Lee, Phys. Rev. D 89, 066007 (2014).

[28] E. Kiritsis, L. Mazzanti and F. Nitti, JHEP 02, 081 (2014).

[29] S. Chakrabortty, S. Chakraborty and N. Haque, Phys. Rev. D 89066013 (2014).

[30] J. Sadeghi, B. Pourhassan and F. Pourasadollah, Eur. Phys. J. C 74, 2793 (2014).

[31] D. Giataganas and H. Soltanpanahi, JHEP 06047 (2014).

[32] J. Sadeghi and F. Pourasadollah, arXiv:1403.2192 [hep-th].

[33] W. Fischler, P. Nguyen, J. Pedraza, W. Tangarife, JHEP 08028 (2014).

[34] J. Boer, V. Hubeny, M. Rangamani and M. Shigemori, JHEP 07, 094 (2009); V. Hubeny and M. Rangamani, Adv. High Energy Phys. 2010, 297916 (2010).

[35] R. P. Feynman and F. L. Vernon, Ann. Phys. 24, 118 (1963); 281, 547 (2000).

[36] J. Schwinger, J. Math. Phys. 2, 407 (1961); L. V. Keldysh, Sov. Phys. JETP 20, 1018 (1965); K. T. Mahanthappa, Phys. Rev. 126, 329 (1962); P. M. Bakshi and K. T. Mahanthappa, J. Math. Phys. 4, 1 (1963); ibid. 4, 12 (1963); K.-C. Chou, Z.-B. Su, B.-L. Hao, and L. Yu, Phys. Rep. 118, 1 (1985); J. Rammer and H. Smith, Rev. Mod. Phys. 58, 323 (1986). 
[37] A. O. Caldeira and A. J. Leggett, Physica A 121, 587 (1983).

[38] H. Grabert, P. Schramm, and G.-L. Ingold, Phys. Rep. 168, 115 (1988).

[39] C. P. Herzog and D. T. Son, JHEP 03046 (2003).

[40] Skenderis K and Rees B C van, Phys. Rev. Lett. 101081601 (2008).

[41] M. Nielsen and I. Chuang, Quantum Computation and Quantum Information, (Cambridge University Press, 2000).

[42] W. H. Zurek, Phys. Rev. D 24, 1516 (1981); ibid 26, 1862 (1982); Phys. Today 44, 36 (1991); W. G. Unruh and W. H. Zurek, Phys. Rev. D 40, 1071 (1989); J. P. Paz, S. Habib and W. H. Zurek, Phys. Rev. D 47, 488 (1993); W. H. Zurek, S. Habib and J. P. Paz, Phys. Rev. Lett. 70, 1187 (1993); W. H. Zurek, Prog. Theor. Phys. 89, 281 (1993).

[43] P. M. V. B. Barone and A. O. Caldeira, Phys. Rev. A 43, 57 (1991).

[44] J.-T. Hsiang and L. H. Ford, Phys. Rev. Lett. 92, 250402 (2004).

[45] J.-T. Hsiang and D,-S. Lee, Phys.Rev. D 73065022 (2006).

[46] J.-T. Hsiang, T.-H. Wu, and D.-S. Lee, Phys. Rev. D 77, 105201 (2008).

[47] J.-T. Hsiang, T.-H. Wu, and D.-S. Lee, Ann. Phys. 327, 522 (2012).

[48] J.-T. Hsiang, T.-H. Wu and D.-S. Lee, Found. Phys. 41, 77 (2011).

[49] A. Chamblin and J Michelson, Class.Quant.Grav. 24, 1569 (2007).

[50] H. Liu, J. McGreevy and D. Vegh, Phys.Rev. D 83065029 (2011).

[51] A. J. Niemi and G. W. Semenoff, Ann. Phys. 152105 (1984); A. J. Niemi and G.W. Semenoff, Nucl. Phys. B 230181 (1984).

[52] C.-H. Wu and D,-S. Lee, Phys.Rev. D 71125005 (2005).

[53] J. Anglin, J. Paz and W. Zurek, Phys. Rev. A 554041 (1997). 